\title{
Conceptual Design of a Hydraulic Valve Train System
}

\author{
J. Pohl, A. Warell, P. Krus, J.-O. Palmberg
}

Variable valve train systems have been brought into focus during recent years as a means to decrease fuel consumption in tomorrow's combustion engines. In this paper an integrated approach, called simulation driven experiments, is utilised in order to aid the development of such highly dynamic systems. Through the use of systematic design methodology, a number of feasible concepts are developed. Critical components are subsequently identified using simulation. In this approach, component behaviour is simulated and validated by measurements on prototype components. These models are unified with complete system models of hydraulically actuated valve trains. In the case of the valve trains systems studied here component models could be validated using comparably simple test set-ups. These models enable the determination of non-critical design parameters in an optimal sense. This results in a number of optimised concepts facilitating an impartial functional concept selection.

Keywords: simulation, simulation based optimisation, conceptual design, valve train system.

\section{Introduction}

In recent years fuel consumption of today's vehicle engines has been brought into focus. One means to increase the overall efficiency is by means of fully variable valve actuation, where valve events and lifts for both intake and exhaust valves can be varied independently. This is especially true for part load operation, where the pumping losses due to the throttle blade can be decreased significantly [1], [4].

Currently, a wide range of systems with varying degrees in actuation flexibility are under development or at a prototype stage. Two different types of actuation devices are already used in production engines of today. Such devices are commonly add-on systems to camshaft based valve actuation. With camphasers, intake and/or exhaust valve overlap can be adjusted, keeping a constant valve duration. For the other type of system, valve actuation is influenced by using two different cam lobes depending on engine speed. With these comparably simple add-on approaches, a significant increase in efficiency can be gained. However, these two actuation principles are not sufficient in order to reduce pumping losses at part load [1].

This paper treats variable valve actuation from a conceptual point of view. The focus is on the actuation system itself rather than the influences on combustion and emissions. The usual procedure for concept validation of variable valve train systems is to build a prototype of the entire system, which can be both costly and time consuming. Such systems are in general difficult to evaluate analytically due to their highly dynamic nature. Using the Simulation Driven Experiments approach presented here, it is possible to identify bottlenecks or components jeopardising a proper system function early in the concept evaluation phase. Using numerical simulation, the valve train system is in consequence decomposed into a few minor prototypes, resulting in a number of less complex experiments. Thus, design cycle time and development cost of new systems are reduced.

\section{Conceptual system design}

The focus of this paper is twofold, that is Simulation Driven Experiments, which will be explained in detail later on, and conceptual design of an electro-hydraulic valve train system. In this case the issue of conceptual design is system design, i.e. the composition of components to a system in order to fulfil a required objective. The conceptual design phase constitutes only a small part of the entire design process but has major influence on the final result. As suggested by Roozenburg and Eekels [6], the conceptual design phase itself can conveniently be broken down into three different tasks.

\subsection{Specification}

The focus of the specification task is to state desired properties and functions of the final product. The requirement specification serves two separate purposes: it provides guidelines for the development process as well as information required for product evaluation. The requirement specification is a working document and is both altered and updated during the entire process. As the product evolves, previously unknown information will be available and must be added to the product specification. Consequently, the specification changes from undetailed and solution independent to specific and solution dependent. The starting-point for a design specification should be the analysis of the product life cycle. Between origination and disposal a product goes through several processes, such as manufacturing, assembly, distribution, installation, operation, maintenance, use, re-use, and disposal. Each of these processes influences the design specification. At the preliminary design stage, it may be impossible to find the answer to all these questions, especially for complex products. This paper focuses on the performance and functional issues of the design specification.

\subsubsection{Performance/function}

- What is the main purpose of the product?

The main purpose of the product is to control the movement of the intake and exhaust valves of an Otto engine, in order to improve overall engine efficiency together with the ability to influence engine emissions.

- What additional function(s) does the product have to fulfil? What are the parameters by which the functional characteristics will be assessed (speed, power, strength...)? 
Valvetrains in production engines of today are camshaft based, which drastically restricts the variability in valve lift, duration and timing. By far, the largest benefit of a fully variable valve train is the ability of non-throttled load control, as explained earlier. Variable valve lift may not be needed at any expense, but may be compensated for by valve deactivation at low engine speeds and loads. Important is the valve seating velocity, as this may not be too high, due to noise and strength reasons.

- What performance properties are required?

The maximum engine speed is restricted to $6000 \mathrm{rev} / \mathrm{min}$ at a valve lift of $8 \mathrm{~mm}$. Valve weight is assumed not to exceed $60 \mathrm{~g}$. The energy consumption of the system should not exceed the energy consumption of present.
- What outer dimensions are desired?

The valve train height must exceed today's systems. A decrease in height is opportune. The width is not as critical as the height, but preferable not larger than today's system.

\subsection{Functional decomposition}

The second task involves an investigation into what the product is meant to do, i.e. to establish the technical process, also called "black-box model" (stating the operand to be transformed, the transformation taking place, and the different states of the process).

Following the functional decomposition a function/ means-tree is developed. In a function/means-tree, the main

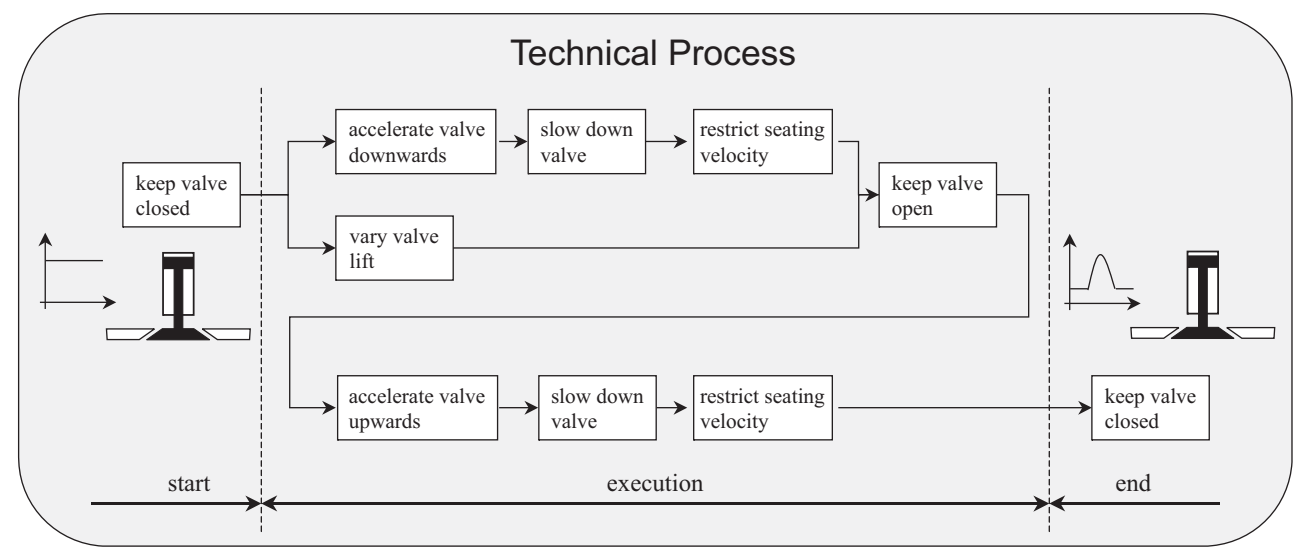

Fig. 1: A technical process model including sub-tasks for the variable valve train

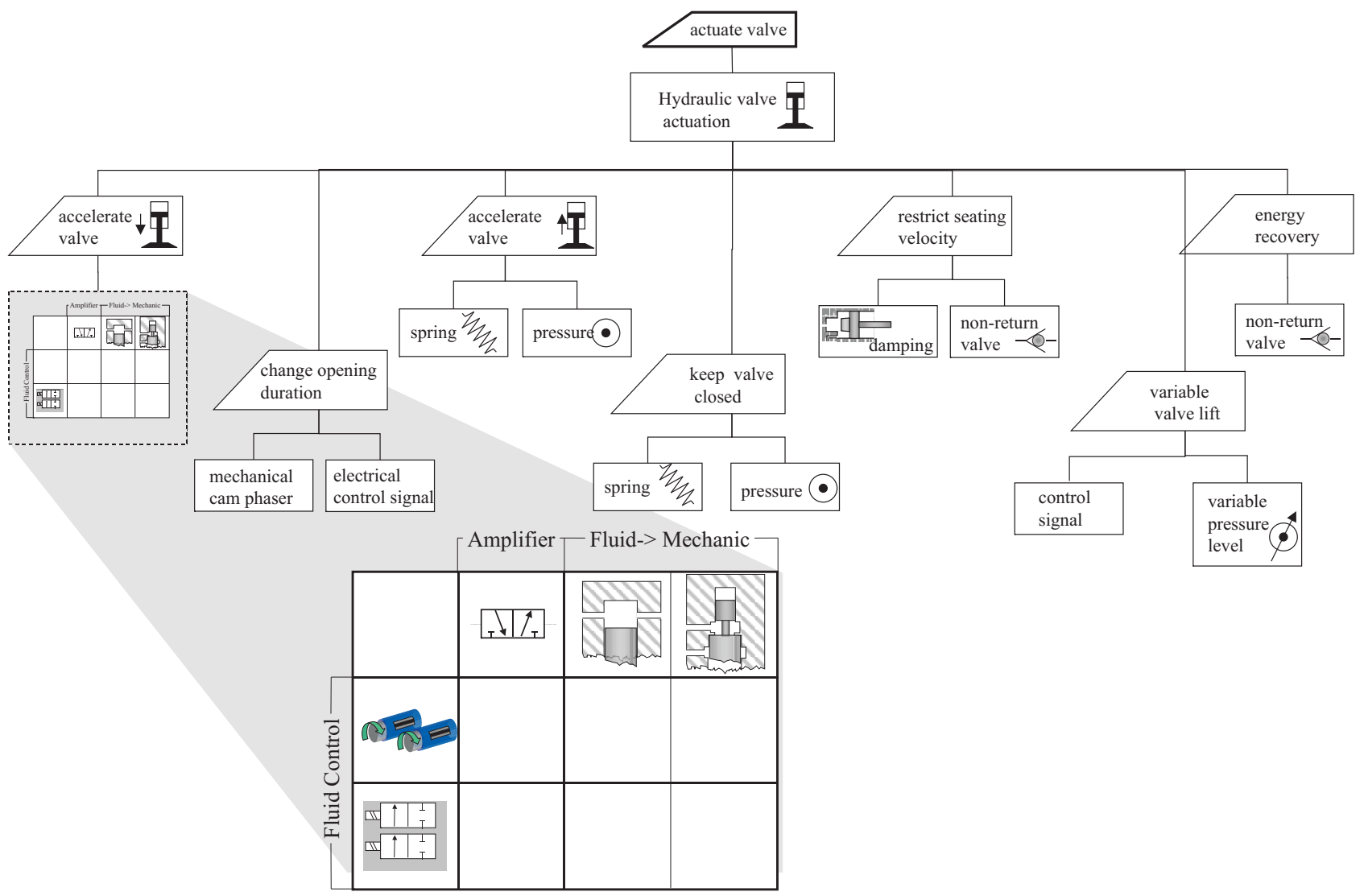

Fig. 2: Function/means-tree developed for the variable valve train system. Sub-functions are derived from the technical process model. 


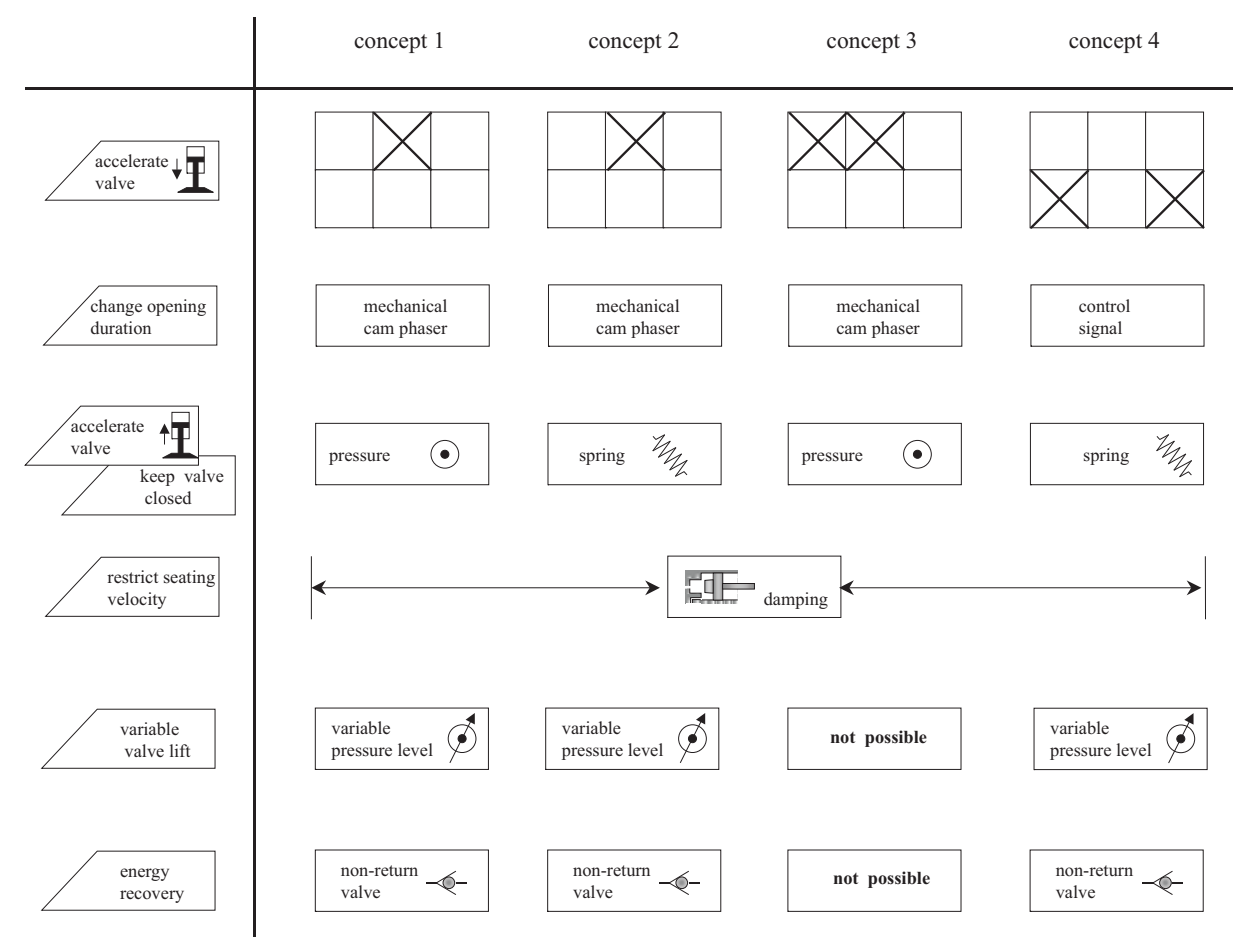

Fig. 3: Table of concepts for the variable valve train system. The means combination matrix was used to represent the alternative means for the "accelerate valve downwards" function.

functions derived from the technical process model are listed together with possible means to realising the required functions. The generation of means is generally regarded as the most demanding phase in conceptual design, since the final solution is only composed of function carriers from the function/means-tree. The function/means-tree is used in order to explore the solution space for all the sub-problems in a systematic manner and to divide it into several distinct classes. A means could be a solution principle, a geometrical model describing a part of the technical system etc. The function/means-tree for the valve train is shown in Figure 2. The sub-functions are extracted from the technical process model and means for these functions are generated. A means combination matrix was utilised in order to represent the combinatory alternatives for the function "accelerate valve downwards". As shown in Figure 2 such a matrix efficiently reduces the size of the function/means-tree.

\subsection{Synthesis}

The final phase is to generate a number of concepts. From the function/means-tree, a morphological matrix containing all generated means to respective functions, is formed. By choosing one function carrier for each function several concepts are generated. Each concept represents a unique combination of means. The aim is now to select the optimal concept for further development, given a set of evaluation criteria. For complex dynamic systems, this evaluation is traditionally done by experiments using a prototype system for each competing concept. However, this procedure is both costly and time consuming.

An alternative evaluation methodology denoted „Simulation Driven Experiments" is therefore proposed in the following section.

\section{The simulation driven experiment approach}

The Simulation Driven Experiments methodology utilises a simulation model of the entire system that delivers results equivalent to the experiment in terms of information needed for concept selection. The overall procedure of the approach can be seen in Figure 4. As opposed to typical industrial practice where simulation is used as an analysis tool rather than a design tool, in this approach the simulation drives the selection of experiments. In other words, the simulation serves the dual purpose of identifying bottlenecks and providing input to the component experiments. In this manner, numerical simulation is used in a first step to identify bottlenecks. Studying the dynamic behaviour of the bottleneck with specialised simulation tools (for instance a CFD-tool for fluid flow calculations) is often time consuming at the conceptual design stage and capturing of the complete dynamic behaviour can not be guaranteed. This necessitates the use of prototyping for validation of component models.

The final aim of the approach presented here is a component model, validated by experiments, that can be used for subsequent evaluation of the overall concept. However, the approach does not specify how the experiment should be conducted in terms of fidelity. Since competing concepts often share identical components, the validated component model can favourably be reused.

Designing the test set-up can also be facilitated by the simulation driven experiments approach. Cause and effect of the test set-up may be altered since a physics based component model is used rather than a black-box model. The task of the valve train system is to accomplish a certain valve opening. This is synonymous with a certain oil flow through, for instance the check valve. Consequently, it is insignificant 


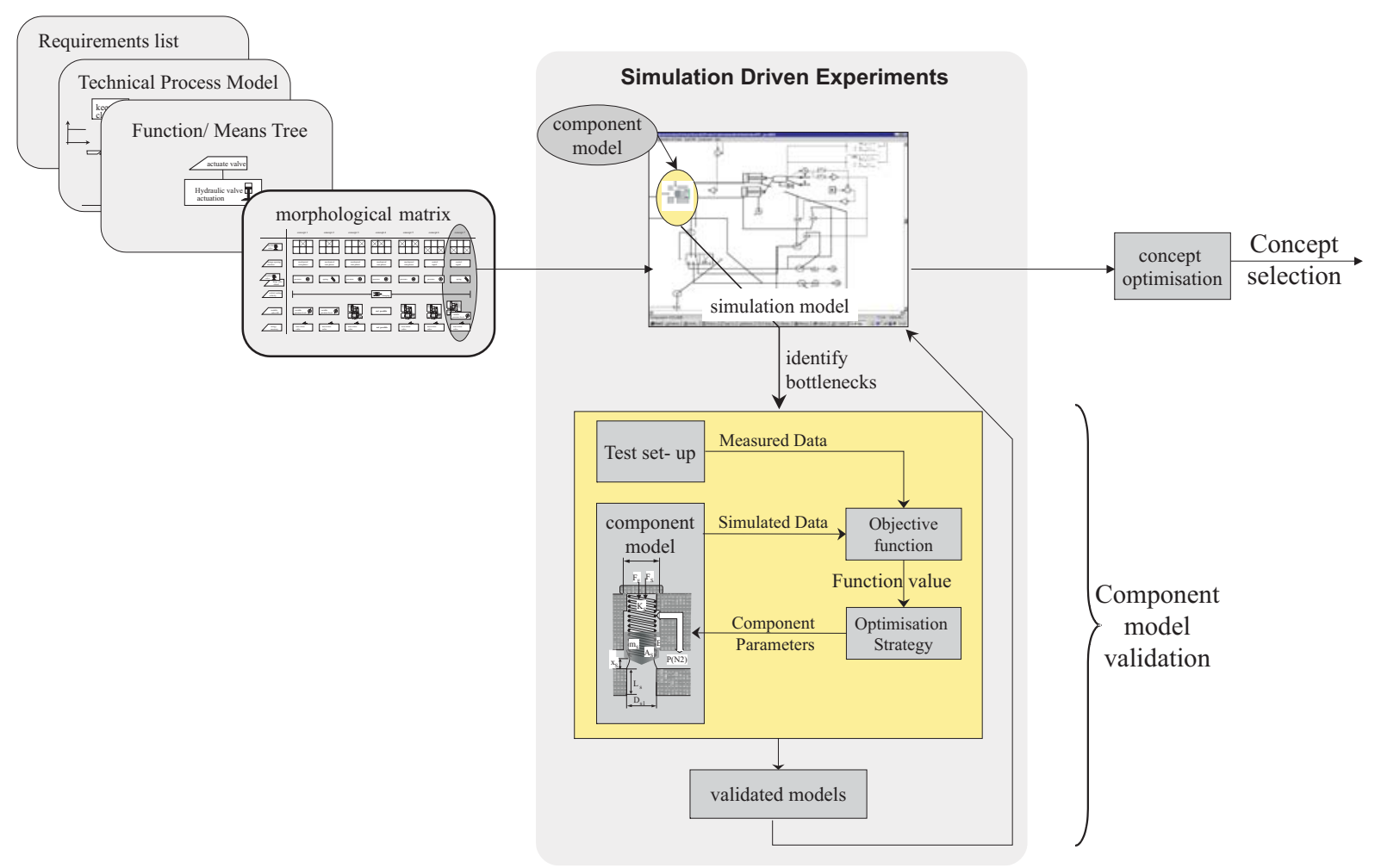

Fig. 4: The overall structure of the Simulation Driven Experiments approach

for the check-valve whether this oil flow is produced by a "real" valve train or any other means.

Model parameterisation can be done with common system identification methods, or even by simulation based optimisation. For the latter approach, the test stand is simulated and optimisation is used to adjust the computed system characteristics to the measured characteristics, see [5]. Thus all state variable of the component need not to be measured, as this can be a quite difficult task. The validated component model is then implemented directly in the overall system simulation model, allowing system performance to be computed.

\subsection{Example 1: Check-valve}

The check valve is an essential component of many hydraulic valve train systems. They are mainly used for energy recovery purposes in order to decrease the valve train's energy consumption. Check valves for fluid power applications usually consist of a spring and a ball in a housing.

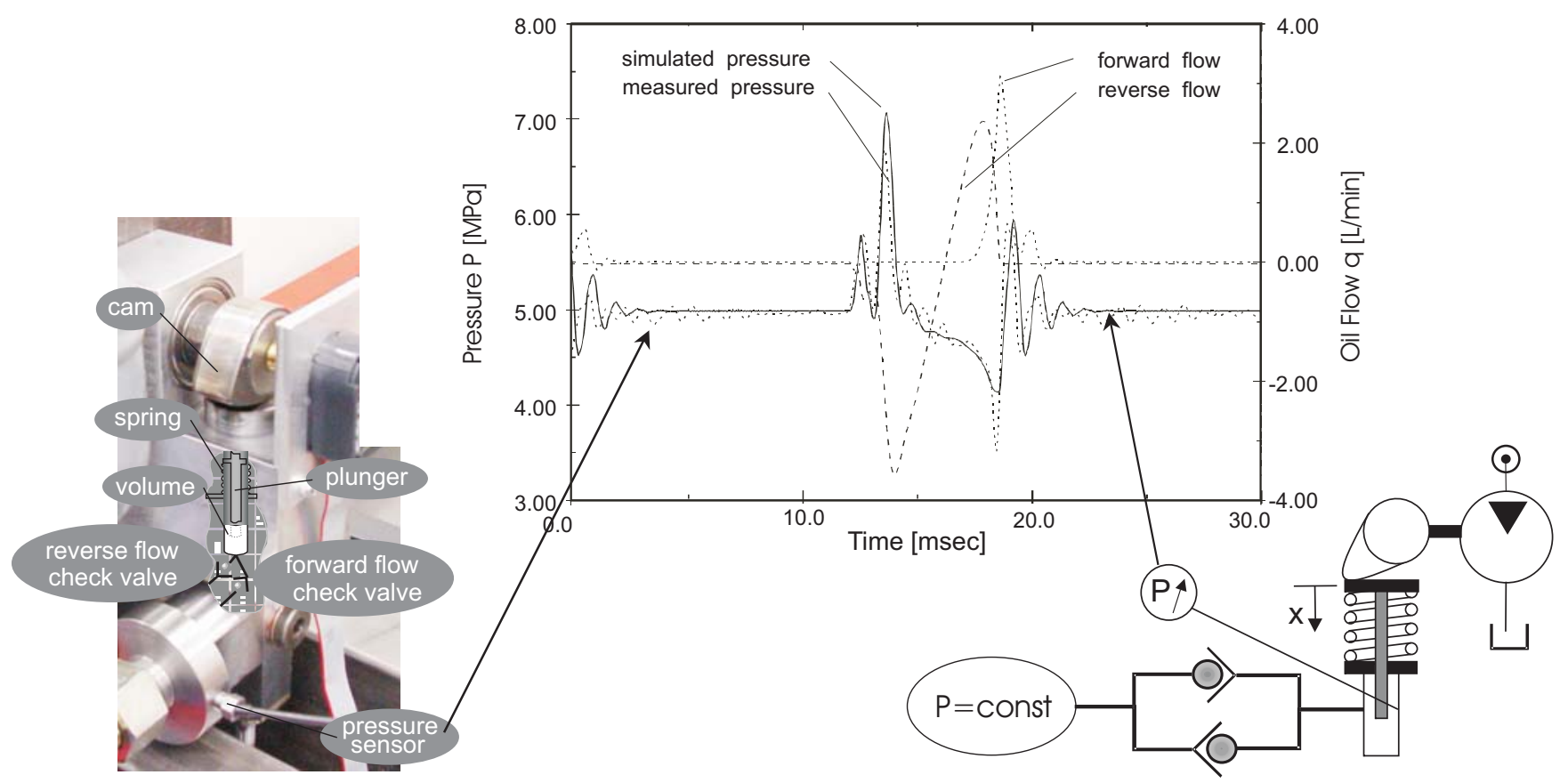

Fig. 5: Simulated and measured pressure signals and corresponding oil flows through the check valves 


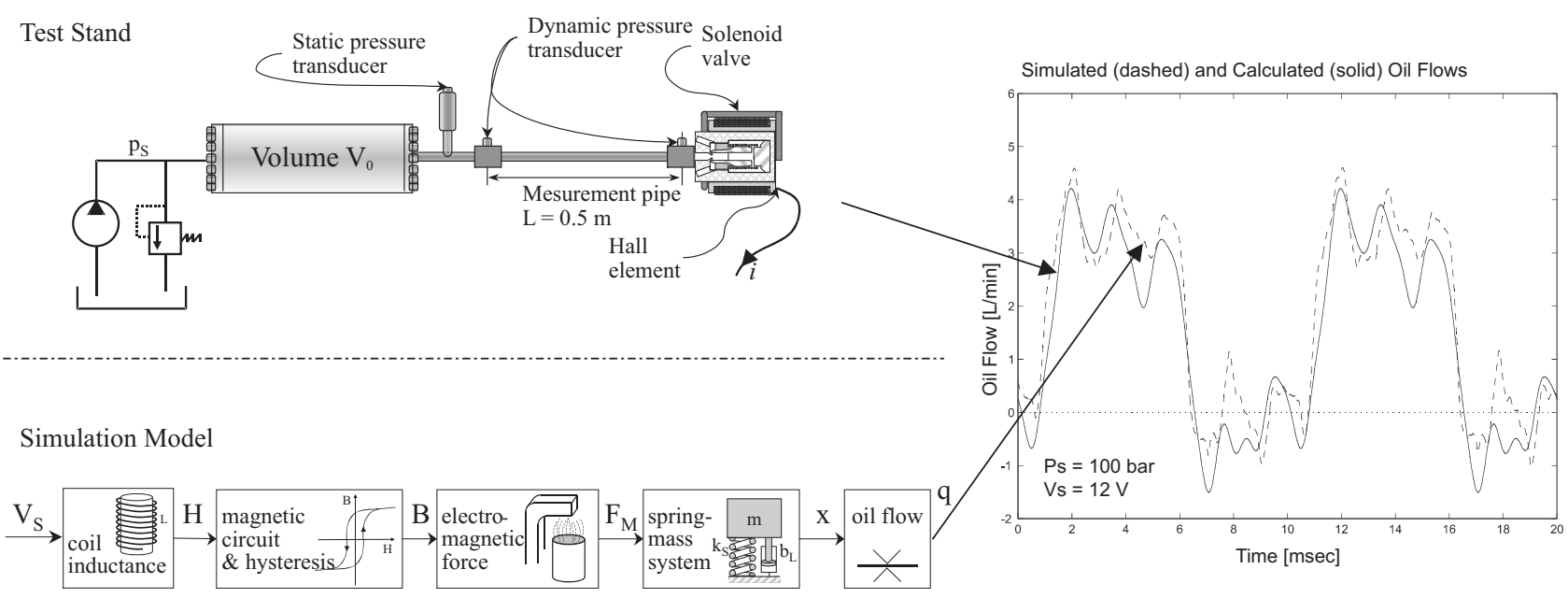

Fig. 6: From the two pressure signals the oil flow was calculated using the 2-microphone method. The dashed line represents the oil flow simulated by the model.

Together with the spring the ball constitutes a $2^{\text {nd }}$ order system, but the break frequency is strongly influenced by the impedance of the oil column in the valve itself, as well as flow forces. Flow forces are usually difficult to obtain from technical component data, which makes complementary tests necessary. The dynamic behaviour of the valves can be studied using a test set-up, such as the one shown in Figure 5.

In the test set-up a cam acts on a plunger moving up- and downwards in an oil volume. During the downwards stroke, oil flows through the forward check valve and during the upwards stroke through the reverse flow valve. By measuring the pressure signal inside the volume, the back-flow through either valve can be estimated. The diagram in Figure 5 shows the measured and calculated pressure signals in the volume together with the corresponding oil flows through the valves. The latter ones were calculated using a simulation model of the test set-up.

\subsection{Example 2: Solenoid-valve}

The solenoid model has to handle the transformation of an input current to an electro-magnetic force on the spool of the valve, covering all the intermediate system states. Material properties such as hysteresis and saturation have shown to be important in solenoid modelling, see [5].

In Figure 6 the oil flow has been calculated with the two-microphone method, see [8]. In this method, two dynamic pressure signals are measured in a specially designed measurement pipe. Together with a description of the pipe in the frequency domain, the oil flow through the pipe in the time domain is calculated. The dashed line represents the simulate oil flow.

\section{Competing valve train concepts}

In order to clarify the approach presented in the previous section, two valve train concepts from the morphological matrix were studied. Characteristics of critical components have been obtained using two specially designed yet simple test set-ups. The validated component models are implemented in the valve train system models to be studied.

By numerical simulation two critical components could be identified, namely the solenoid and the check valves. According Figure 4 these models are implement in the overall simulation models of the two valve train concepts in question

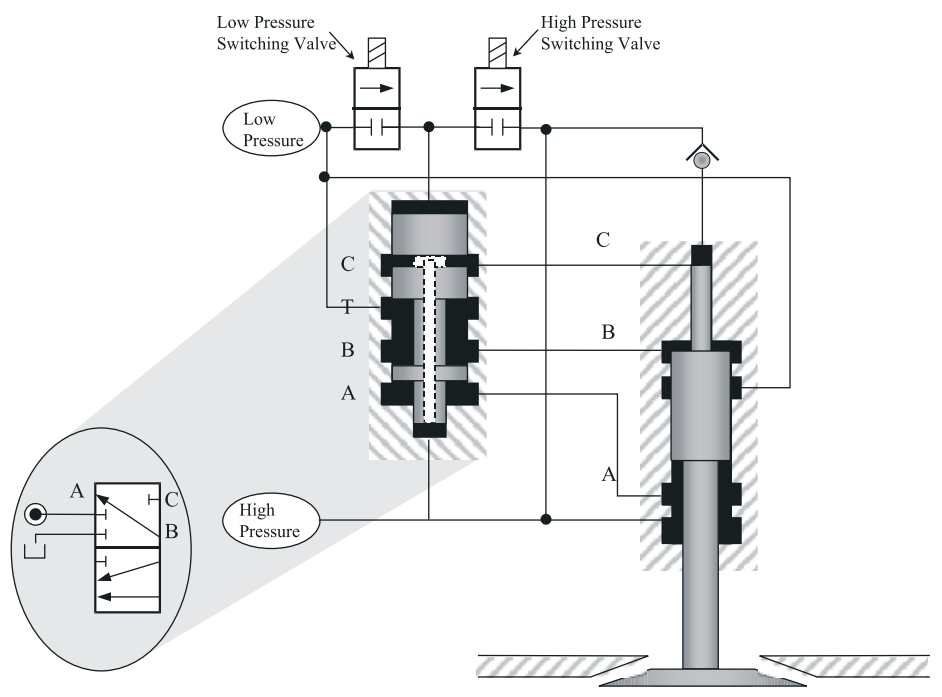

Fig. 7: The FluMeS valvetrain system 
a system that has been developed by the Ford Motor Company [7] and a concept developed by the Division of Fluid and Mechanical Engineering Systems (FluMeS) at Linköping University. Figure 7 shows the principal layout of the FluMes-system. The modelling and simulation of both these systems was done in the Hopsan simulation package [3], developed at the same division.

The valve train system consists of a hydraulic piston which is mounted directly onto the engines valve stem. The oil flow is controlled by a hydraulic main stage working as a flow amplifier since the flow capacity of the solenoid valves itself is not sufficient. If there should exist fast switching valves with sufficient flow capacity in future, this main stage can be omitted. In this respect, the valve train system presented constitutes a very flexible solution.

The valve train system works as follows: A valve opening is initiated by the high-pressure solenoid valve, so that the main stage is switched to its lower position. In this manner, the A and $\mathrm{B}$ port of the hydraulic piston will be connected and oil is flowing from the high pressure side through the lower piston chamber in to the upper piston chamber. As a consequence, the engine valve moves downwards. After approximately half the strokelength the piston cut the oil supply from the high pressure side and a connection to the low pressure side is established, see Figure 7. Due to the valves inertia it will continue to move downwards until the kinetic energy has been used to draw oil from the low pressure side into the upper piston chamber. During the entire downwards stroke, oil from the high pressure side flows through the check-valve. The advantage with this type of valve train system is that the hydraulic piston remains due to the check-valve in the extend position irrespective of the solenoid valves state. In this manner, the engine valves movement is only initiated by the solenoid valve which may be activated longer than the actual engine valve stroke. Thus solenoid valves closing characteristic is not critical a critical parameter.

The engine valves closing is initiated by the low pressure switching valve which make the main stage move into the upper position. Both B an $\mathrm{C}$ port are connected to tank.

In Figure 8 the basic layout of Fords system is shown, but unlike the system presented in [7] a main stage for flow amplification purposes as in the FluMeS-system has been added. In this study the Ford system is meant to use the same solenoid- and check-valves as the system in Figure 7 in order to make concept selection be based on similar premises. In this system, the downward stroke is initiated with the high pressure-switching valve.

Such a system consists of two switching and two check valves as well as a hydraulic piston that is mounted directly on the engine valve stem. In this system, the downward stroke is initiated with the high pressure-switching valve. The valve is closed after a certain distance, about half the stroke distance, and oil is sucked from the low-pressure side via the corresponding check valve. The engine valve is thus retarded and finally reaches the stroke length. The upward stroke is a reversed procedure of the downward stroke, initiated via the

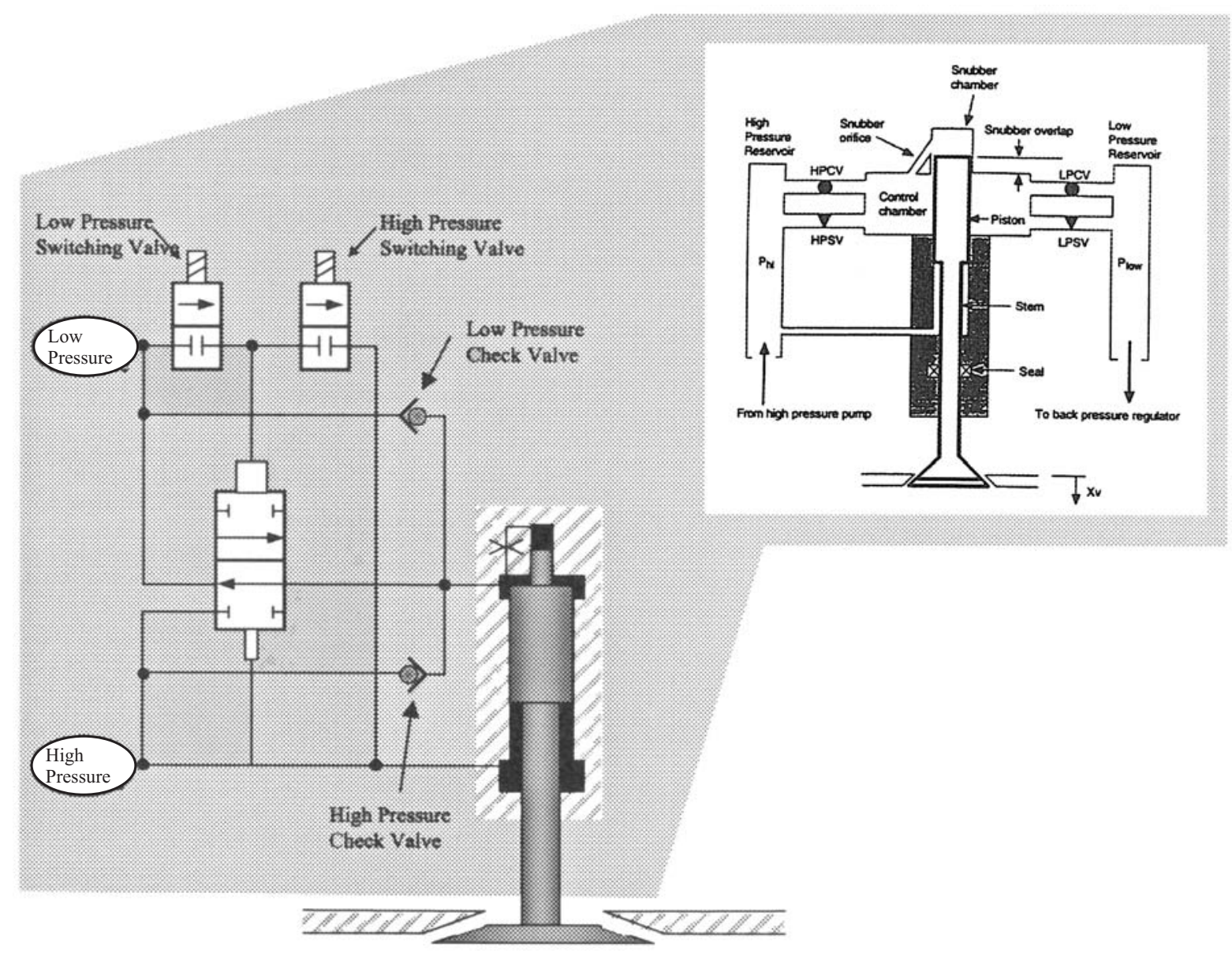

Fig. 8: Ford solution to the valve train problem. In the background a drawing of the concept from [7] is shown. 


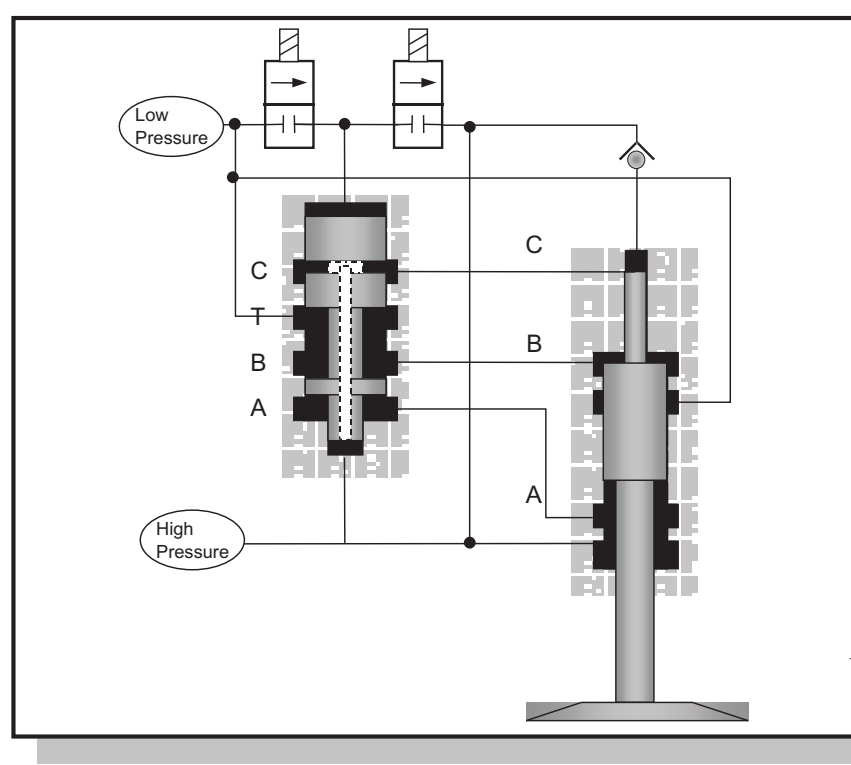

Simulation

Fig. 9: Principal procedure for simulation based optimisation

low-pressure switching valve but here retardation is done by feeding back high-pressure oil into the high-pressure line. In this manner, the high- and low-pressure checkvalves are vital components to reduce the energy consumption of the system, as valuable high-pressure oil only is used during a fraction of the engine valve stroke.

\section{Concept optimisation}

According to the process map shown in Figure 4 concept optimisation should be conducted before proceeding to the concept selection stage. In Figure 9 is shown how optimisation based on numerical simulation can be done. Unlike classic mathematical optimisation problems, the objective function is here not given directly, rather than as a result from a simulation. In this manner the simulation model is used in order to produce the input data to the objective function that is used by the optimisation strategy in order to do a ranking between different parameter sets.

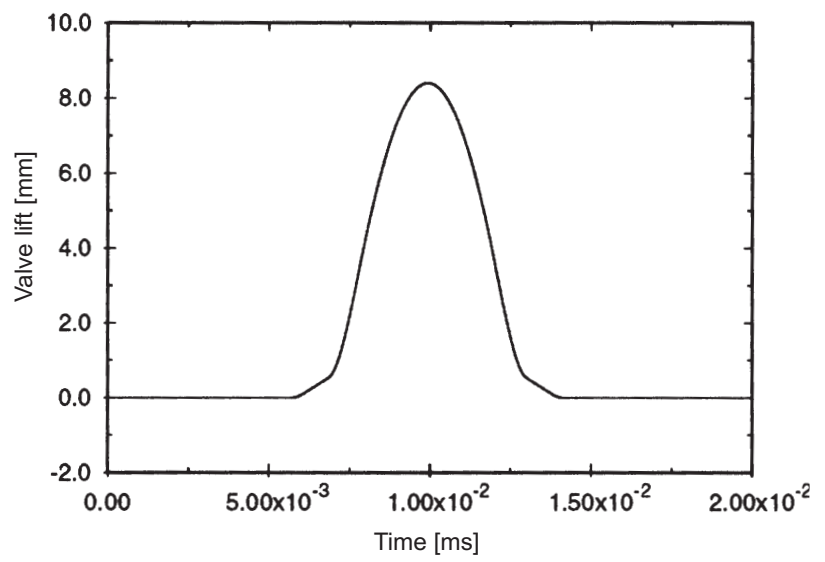

Fig. 10: The desired valve lift as a time function for an engine speed of $6000 \mathrm{rpm}$
To formulate an objective function can be a quite difficult task when several objectives are to be met. In this case the valve train system has to actuate the engine valve according to a certain cam profile while at the same time keeping the energy consumption low. The cam profile in question is shown in Figure 10 and is a standard cam profile as it is used in many of today's production engine. The valve lift is here shown as a time function for an engine speed of $6000 \mathrm{rpm}$.

Figure 11 shows the valve opening of the FluMeS-system for a given input signal obtained by optimisation. The correspondence between the pattern valve opening and the simulated one (dashed) is quite good.

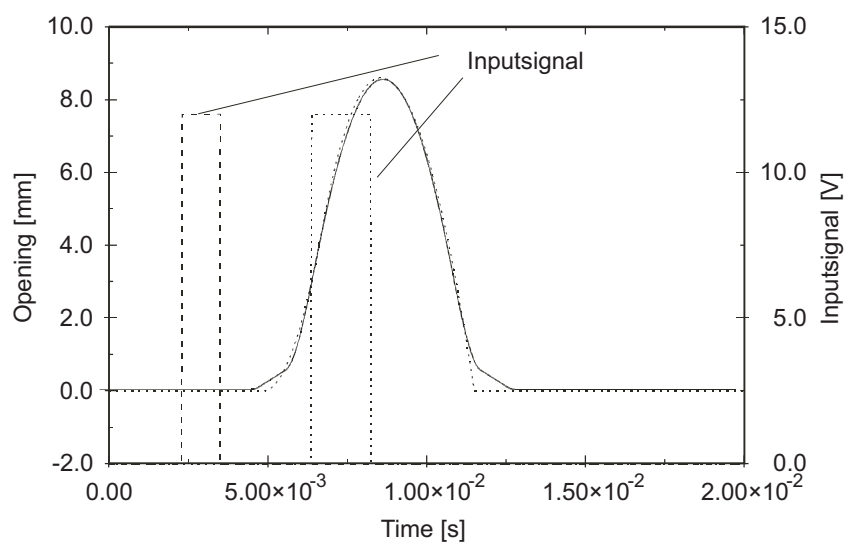

Fig. 11: Simulated (dashed) and given valve opening for the FluMeS-system as well as input signals to the solenoid valves for an engine speed of $6000 \mathrm{rpm}$

In Figure 12 the opening of check-valve, main stage and solenoid valves is shown (left diagram) as well as the oil flow from the high pressure side and check-valve together with the valve opening. The High pressure oil flow per engine cycle is a measure for the energy consumption of the system. 

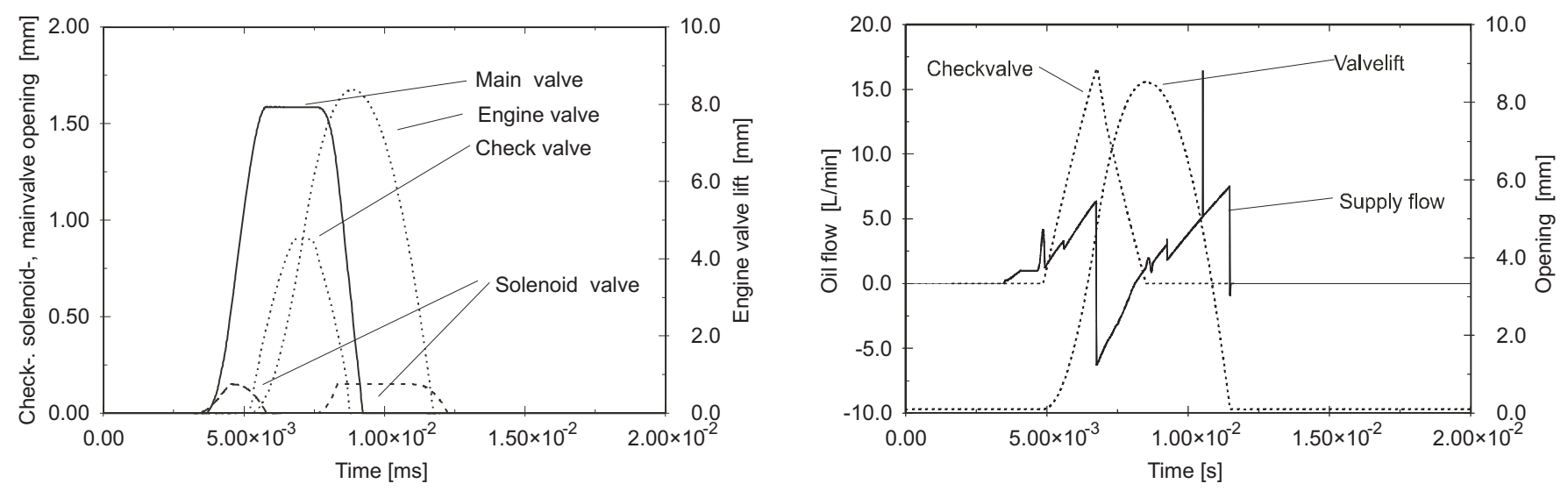

Fig. 12: Opening of check-valve, main stage and solenoid valves and corresponding oil flows for the FluMeS-system

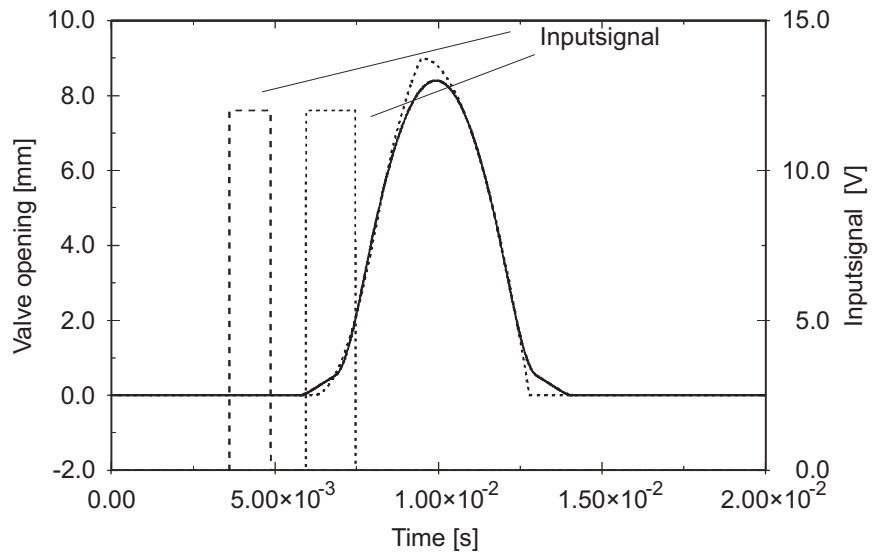

Fig. 13: Simulated (dashed) and given valve opening for Fords-system as well as input signals to the solenoid valves for an engine speed of $6000 \mathrm{rpm}$

In Figure 13 the valve opening together with the input signals to the solenoid valves is chosen in case of the Ford system. The capacity of this system to follow a certain opening profile is obviously worse than for the other concept. However, most important for a valve train system is that opening and closing times are prescribed, the opening and closing gradients s of secondary importance.

In Figure 14 the valve opening for check-valves, solenoid valves and main stage is shown. To the left the corresponding oil flows are shown.

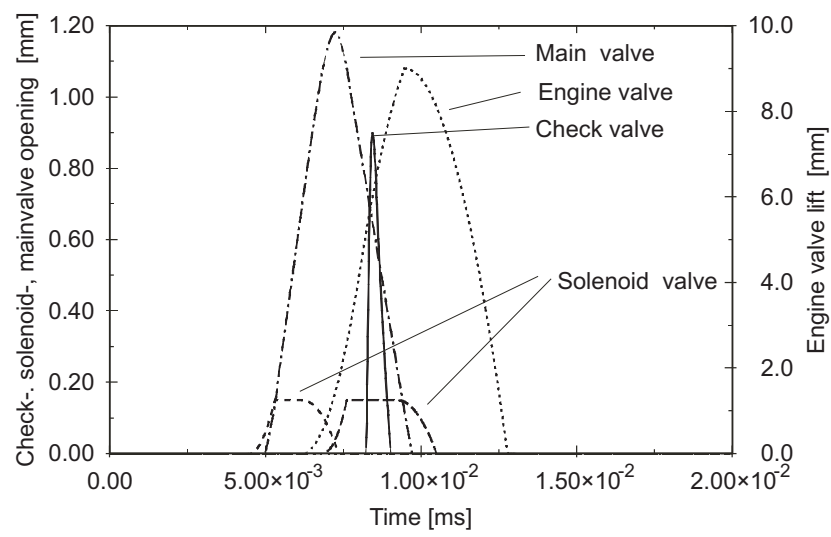

The FluMeS-system is more suitable when a certain opening profile of the engine valves is required as can be seen from Figure 11 and 13. This is mainly due to the solenoid valve used in this study. In the case of the Ford-System, both opening and closing characteristics of the solenoid valve influence the engine valve opening. For the FluMeS-system the solenoid valve closing is irrelevant, as this is handled by internal groves of the valve piston.

The energy recovery function as it is presented in [7] is compared with hydraulic pendulum, where kinetic energy is transformed into potential energy, and vice versa. This demands for very fast solenoid valves for higher engine speeds, as these must activate the flow amplifying main stage sufficiently fast in order to make energy recovery possible on the downwards stroke. In this study the high pressure solenoid valve was not in use (see Figure 15) which is synonymous with that energy recovery was not possible on the downwards stroke.

The energy consumption for both the Ford and FluMeS system lies around $3 \mathrm{kw}$ for an engine with 20 valves. This was without any force due to combustion, this may make an increase in system pressure necessary, which will raise energy consumption. From a design and manufacturing point of view the Ford system appears to be the simpler design solution, as the main stage is kept simpler and the cylinderhead design becomes simpler due to less channels. On the contrary the other system is, as already explained earlier, not dependent on the closing characteristics of the solenoid valves. Closing is

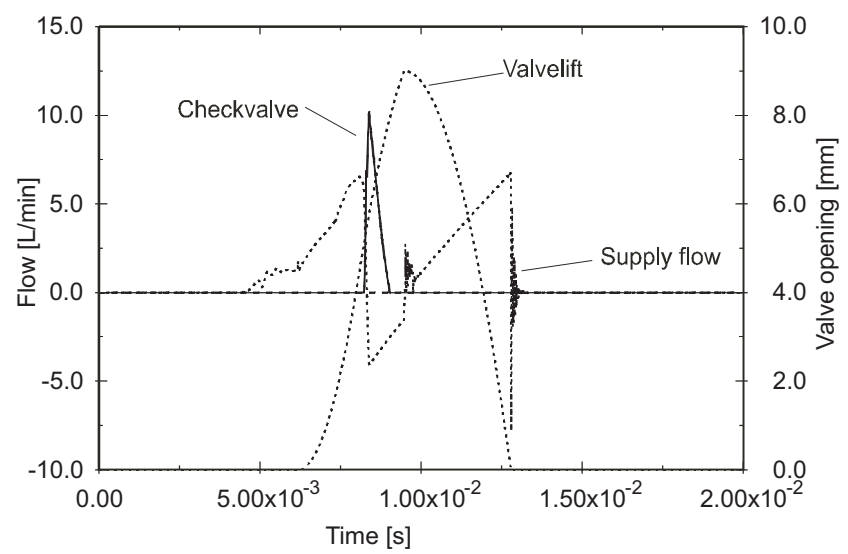

Fig. 14: Opening of check-valve, main stage and solenoid valves and corresponding oil flows for Fords-system 
for many solenoid valve designs guaranteed by a spring force. If the valve is not fully pressure balanced, the closing sped may be pressure dependent. This makes the control strategy more difficult in case of the Ford system.

\section{Conclusions}

The purpose of this work is twofold. First it shows how conceptual design techniques can be used in variable valve train development. Secondly, the feasibility of the Simulation Driven Experiments approach to be used in conceptual design is studied. Simulation is used in order to both identify critical components in a valve train system and for model adaptation together with optimisation. The component models which have been validated against measured data are fed back into the overall simulation model. This significantly raises the confidence level of the overall simulation model.

In this paper, two competing valve train concepts, developed using well known conceptual design techniques, were studied. Both systems were optimised according to exactly the same objective function. Thus the objective function value is a direct measure for system performance. Here energy consumption and the ability to provide certain valve opening characteristics were taken as quality criteria. In this manner, a functional concept selection can take place more impartially.

As demonstrated in this paper the use of the Simulation Driven Experiments approach may significantly reduce the amount and the need of full-scale hardware testing. The approach shows significant potential for industry in terms of reduction of design cycle time and development costs.

\section{References}

[1] Adamis, P., Gnegel, P.: Variable Ventilsteuerung und deren Einfluss auf Verbrauch und Emissionen. VDI Berichte Nr. 1170, Verien Deutscher Ingenieure, Germany, 1994

[2] Heywood, J. B.: Internal Combustion Engine Fundamentals. McGraw Hill, 1988

[3] Hopsan: Hopsan, a Simulation Package, User's Guide. Technical Report LITH-IKP-R-704, Division of Fluid and Mechanical Engineering Systems, Linköping University, Sweden, 1991

[4] Miller, R. H., Davis, G. C., Newman, C. E., Levin M. B.: Unthrottled Camless Valvetrain Strategy for Spark-Ignited Engines. 1997 Fall Technical Conference, ASME, USA, 1997

[5] Pohl, J., Sethsson, M., Krus, P., Palmberg, J.-O.: Modelling and Simulation of a Fast 2/2 Switching Valve for Hydraulic Valve Train Applications. LiTH-IKP-R1135, Division of Fluid and Mechanical Engineering Systems, Linköping University, Sweden, 2000

[6] Roozenburg, N. F. M., Eekels, J.: Product Design: Fundamentals and Methods. John Wiley \& Sons, 1995

[7] Schlechter, M. M., Levin, M. B.: Camless Engine. SAE Paper 960581, Society of Automotive Engineers, USA, 1996

[8] Weddfelt, K.: On Modelling, Simulation and Measurements of Fluid Power Pumps and Pipelines - with special Reference to Flow Pulsations. Dissertation, Linköping University, Sweden, 1992

Dr Jochen Pohl

e-mail:jpohl12@volvocars.com

Prof. Petter Krus

Prof. Jan-Ove Palmberg

phone: +4613281198

fax: +4613130414

Fluid and Mechanical Engineering Systems

Linköping University

S-581 83 Linköping, Sweden

Lic.-Eng. Anders Warell

Industrial Design Engineering

Chalmers University of Technology

S-412 96 Göteborg, Sweden 